\title{
Is cancer a pure growth curve or does it follow a kinetics of dynamical structural transformation?
}

\author{
Maraelys Morales González ${ }^{1}$ Javier Antonio González Joa², Luis Enrique Bergues Cabrales ${ }^{3 *}$, \\ Ana Elisa Bergues Pupo ${ }^{4}$, Baruch Schneider ${ }^{5}$, Suleyman Kondakci ${ }^{6}$, Héctor Manuel Camué Ciria ${ }^{3}$, Juan Bory Reyes ${ }^{7}$, \\ Manuel Verdecia Jarque ${ }^{8}$, Miguel Angel O'Farril Mateus ${ }^{9}$, Tamara Rubio González ${ }^{10}$, \\ Soraida Candida Acosta Brooks ${ }^{11}$, José Luis Hernández Cáceres ${ }^{12}$ and Gustavo Victoriano Sierra González ${ }^{13}$
}

\begin{abstract}
Background: Unperturbed tumor growth kinetics is one of the more studied cancer topics; however, it is poorly understood. Mathematical modeling is a useful tool to elucidate new mechanisms involved in tumor growth kinetics, which can be relevant to understand cancer genesis and select the most suitable treatment.

Methods: The classical Kolmogorov-Johnson-Mehl-Avrami as well as the modified Kolmogorov-Johnson-MehlAvrami models to describe unperturbed fibrosarcoma Sa-37 tumor growth are used and compared with the Gompertz modified and Logistic models. Viable tumor cells $\left(1 \times 10^{5}\right)$ are inoculated to 28 BALB/C male mice.

Results: Modified Gompertz, Logistic, Kolmogorov-Johnson-Mehl-Avrami classical and modified KolmogorovJohnson-Mehl-Avrami models fit well to the experimental data and agree with one another. A jump in the time behaviors of the instantaneous slopes of classical and modified Kolmogorov-Johnson-Mehl-Avrami models and high values of these instantaneous slopes at very early stages of tumor growth kinetics are observed.

Conclusions: The modified Kolmogorov-Johnson-Mehl-Avrami equation can be used to describe unperturbed fibrosarcoma Sa-37 tumor growth. It reveals that diffusion-controlled nucleation/growth and impingement mechanisms are involved in tumor growth kinetics. On the other hand, tumor development kinetics reveals dynamical structural transformations rather than a pure growth curve. Tumor fractal property prevails during entire TGK.
\end{abstract}

Keywords: Fibrosarcoma Sa-37 tumor, Diffusion-controlled nucleation/growth mechanisms, Impingement mechanisms, Isothermal dynamical structural transformation

\section{Background}

Asymptotic growth indicates that a system shifts from positive feedback (which generates exponential growth) to negative feedback (which produces stabilizing growth). This shift is known as sigmoidal ("S-curve" or S-shaped growth). Systems that exhibit S-shaped growth-time behavior are characterized by constraints or limits to growth, as sickle cell disease [1], tumors [2], bacteria and microorganisms [3], among others. Other systems produce Sshaped transformation-time behavior, as crystals $[4,5]$.

\footnotetext{
* Correspondence: berguesc@yahoo.com

${ }^{3}$ Research and Innovation Department, Oriente University, National Center of Applied Electromagnetism, Ave. Las Américas, Santiago de Cuba 90400, Cuba

Full list of author information is available at the end of the article
}

Tumor growth kinetics (TGK) is not well understood so far. TGK has three well-defined stages: the first (Lag stage) is associated with the establishment of the tumor in the host. The second (Log or exponential stage) is related to rapid tumor growth. The third (Stationary stage) shows slow tumor growth asymptotically converging to a final volume [2]. It is expected a fourth stage (Death stage) of TGK, in which tumor dies because the nutrients are depleted by anorexia of animal or human host, showing a decline. This fourth stage is not considered in TGK due to ethical considerations $[6,7]$. In mice, tumor burden should not usually exceed $10 \%$ of the host animal's normal body weight [6]. 
During the last decades, tremendous efforts have been made by both experimentalists and theoreticians to search a suitable growth law for tumors, one of the most striking and interesting issues in cancer research [2, 8-11]. The Logistic equation has been used to describe TGK and the interactions among different competing populations with and without an external perturbation [12, 13]. The Logistic and von Bertalanffy equations have been reported to provide excellent fits for patients and mice bearing tumors, respectively [8]. In contrast, Marušic et al. [9] and Miklavčič et al. [11] show that the standard Gompertz model outperforms both Logistic and von Bertalanffy models. Marušic et al. [9] explain this disparity because the fit is dependent on the applied least squares fitting method. The Gompertz model is the most used to describe TGK $[2,8,10,11,14]$.

The standard Logistic equation (Eq. 1) and the standard Gompertz equation (Eq. 2) are given by [8-11]:

$$
\begin{aligned}
& V(t)=\frac{K^{*} V_{o} e^{r^{*} t}}{K^{*}+V_{o}\left(e^{r^{*} t}-1\right)} \\
& V(t)=V_{o} e^{\left(\frac{\alpha}{\beta}\right)\left(1-e^{-\beta t}\right)}
\end{aligned}
$$

where $V(t)$ represents the untreated tumor volume at time $t$ and $V_{o}$ its initial volume at the beginning of observation $(t=0)$. Experimentally, $V_{o}$ (reached in a time $t_{o}$ ) is any tumor volume that satisfies the condition $V_{o} \geq V_{\text {meas }}$. $\mathrm{V}_{\text {meas }}$ is the minimum measurable tumor volume and reaches in a time, $t_{\text {meas }}$. The constant $r$ " defines the growth rate and $K^{\prime \prime}$ is the carrying capacity $[8,12]$. The parameter $\alpha$ is the intrinsic growth rate of the unperturbed tumor related to the initial mitosis rate. The parameter $\beta$ is the growth deceleration factor related to the endogenous antiangiogenesis processes $[11,15]$ by an overexpression of different antiangiogenic molecules (i.e., Angiostatin, Thrombospondin-1 molecules) $[15,16]$. As tumors are not perturbed with an external agent, this parameter $\beta$ is not related to therapy-induced antiangiogenis [12]. Despite the interpretation of the parameter $\beta$, authors of this study believe that this parameter may be related to other endogenous antitumor processes, as cellular death processes (apoptosis, necrosis, metastasis and exfoliation) and interactions between tumor cells and immune cells [17]. Further experiments are required for a correct interpretation of this parameter.

An important part of tumor vital cycle has already happened long before $V_{\text {meas }}$ is reached [17] and therefore it cannot be described with the Eqs. (1) and (2). However, this part of TGK may be fitted if an effective delay time ( $\tau$ ) is introduced in the Eqs. (1) and (2) [2, 18-20]. Besides, $\tau$ has been included in these two equations to describe Lag stage of bacteria- and microorganism growths [3]. $\tau$ has a crucial role in the modeling of biological processes [21]. The interesting question is if in the case with delay the Logistic model, named modified Logistic model (Eq. 3), or the Gompertz model, named modified Gompertz model (Eq. 4), is the best one for describing early tumor growth as it is believed in the case without delay (Eqs. 1 and 2). Equations (3) and (4) result of the substitution of $t$ by $(t-\tau)$ in the Eqs. (1) and (2):

$$
\begin{aligned}
& V(t-\tau)=\frac{K^{*} V_{\tau} e^{r^{*}(t-\tau)}}{K^{*}+V_{\tau}\left(e^{r^{*}(t-\tau)}-1\right)} \\
& V(t-\tau)=V_{\tau} e^{\left(\frac{\alpha}{\beta}\right)\left(1-e^{-\beta(t-\tau)}\right)}
\end{aligned}
$$

where $\mathrm{V}(\mathrm{t}-\mathrm{\tau})$ represents tumor volume at time $(\mathrm{t}-\mathrm{\tau})$, meaning that the growth at present time $t$ depends on the previous time (t- $\tau$ ). Parameters $\tau$ and $V_{\tau}$ are the time and tumor volume corresponding to inflection point of TGK, respectively. Parameters $r^{*}, K^{*}, \alpha$ and $\beta$ have been defined above in Eqs. 1 and 2.

Different findings have been documented in cancer, as: heterogeneity, anisotropy, fractal property, stiffness, surface roughening, curved surface, high macroscopic shear elastic modulus, among others [17, 21-25]. These findings have been also reported in crystals, despite noticeable differences between tumors and crystals, and in their growth mechanisms [26-30].

The classical Kolmogorov-Johnson-Mehl-Avrami model, named KJMA model (Eq. 5), and modified KolmogorovJohnson-Mehl-Avrami model, named mKJMA model (Eq. 6), have been used to fit entire sigmoidal curve of a crystal [26], given by

$$
\begin{aligned}
& p(t)=1-e^{-(K t)^{n}} \\
& p(t)=1-\left[1+(\lambda-1)(K t)^{n}\right]^{-1 /(\lambda-1)}
\end{aligned}
$$

With

$$
K(T)=K_{o} e^{-E_{a} / R T} \text { (Arrhenius equation) }
$$

where $\mathrm{p}(\mathrm{t})$ is the transformed fraction at $\mathrm{t}$ (fraction of grains that is transformed to crystal phase). $n(n \geq 0)$, $K(T), \lambda(\lambda \geq 1), K_{o}, E_{a}, R$ and $T$ are the Avrami exponent, specific rate of transformation process that depends on temperature, impingement factor, the pre-exponential factor, effective (overall) activation energy of the transformation (or activation energy barrier to crystal formation), Boltzmann constant and temperature, respectively. RT represents the thermic kinetics energy. Arrhenius Eq. (7) is substituted in the Eqs. (5) and (6) to know $\mathrm{K}_{\mathrm{o}}$ and $\mathrm{E}_{\mathrm{a}}$.

In crystals, $\mathrm{K}$ is constant, proportional to the transforming volume/surface area and results of unbalanced diffusion processes (linked to heterogeneity). $\lambda$ represents impingement mechanisms, as: capillarity effect, 
interfacial and superficial phenomena, among others. $\mathrm{n}$ is closely related to nucleation mechanisms, the existence of a lag stage, anisotropy, structural changes, vacancy annihilation, stiffness, surface roughening, curved surface, change of shape and high macroscopic shear elastic modulus of the forming and growing crystal. Additionally, $\mathrm{n}$ is inversely proportional to fractal dimension of the crystal. $n \geq 3$ has been related to spherical shape of crystals, formation of micro-clusters of crystal seeds, high anisotropy and higher vacancies number [26-30].

On the other hand, nucleation and impingement mechanisms emerge to eliminate high energetic instabilities (by thermal fluctuation) during forming and growing crystal structure. Nucleation sites (or vacancy numbers) disorder the interior of forming and growing system and need be filled to guarantee their stability and growth. Deviation from integer value for $\mathrm{n}$ has been explained as simultaneous development of two (or more) types of crystals, or similar crystals from different types of nuclei (sporadic or instantaneous). Nucleation is either instantaneous, with nuclei appearing all at once early on in the process, or sporadic, with the number of nuclei increasing linearly with time [26-30].

KJMA and mKJMA models are phenomenological and not valid when $T$ varies with time [31]. Furthermore, they are developed for the kinetics of phase changes to describe the rate of transformation of the matter from an old phase to a new one, taking into account that the new phase is nucleated by germ nuclei that already exists in the old phase. The Eq. (6) can be reduced to the Eq. (5) when $\lambda$ tends to 1. Wang et al. [26] report that KJMA model cannot be applied to crystal growth when $\lambda>1$ because there are phenomena (i.e., capillarity effects, vacancy annihilation, blocking due to anisotropic growth) that may cause violations to KJMA. Consequently, a misinterpretation of the kinetics may be given if these phenomena are ignored.

We are not aware that KJMA model and mKJMA model have been used to describe TGK. Nevertheless, in principle, these two models can be used to fit Sshaped growth of tumors, taking into account that "S-curve" is universal, the Eqs. (1, 2, 3, 4, 5 and 6) are phenomenological and the above-mentioned findings are common for both tumors and crystals. The application of the Eqs. (5) and (6) may reveal whether other findings not yet described are involved in TGK. Elucidating underlying mechanisms in entire TGK is of great importance for both understanding and planning antitumor therapies. The aim of this paper is to use, for the first time, KJMA and mKJMA models to describe the untreated fibrosarcoma Sa-37 TGK. Also, KJMA and mKJMA models are compared with modified Gompertz and Logistic models.

\section{Methods \\ Mice}

Twenty eight male (6-7 week, 18-20 g) BALB/c mice are studied. Animals are purchased from the National Center for Laboratory Animals Production (Havana, Cuba), housed in clear standard polycarbonate cages of $206 \mathrm{~mm}^{2} \times 12 \mathrm{~cm}$ (4 animals/cage) with hard woodshavings as bedding and given pellet $\mathrm{BALB} / \mathrm{c}$ mice diet and tap water (sterilized and non-chemically treated) ad libitum under controlled environmental conditions, including a temperature of $23 \pm 1{ }^{\circ} \mathrm{C}$ (Sattigungs thermometer of precision $\pm 1{ }^{\circ} \mathrm{C}$, Germany), a relative humidity of $55 \pm 5 \%$ (Fischer Polymeter of precision $\pm 1 \%$, Germany), and a 12-h light/darkness cycle (lights on 7:00-19:00). Bedding and pellets are sterilized by autoclaving. They are changed daily. During the experiment the animals are firmly fixed on plastic boards and show uneasy and quick breathing during fixation. Survival checks for morbidity and mortality are made twice per day. Any animal found dead or moribund is subjected to gross necropsy.

\section{Tumor cell lines}

Fibrosarcoma Sa-37 cell lines are received from the Center for Molecular Immunology (Havana, Cuba). Fibrosarcoma Sa-37 ascitic tumor cell suspensions, transplanted to the $\mathrm{BALB} / \mathrm{c}$ mouse, are prepared from the ascitic form of the tumors. Subcutaneous tumors located in the right flank of the dorsolateral region of mice are initiated by the inoculation of $1 \times 10^{5}$ viable tumor cells in $0.2 \mathrm{ml}$ of $0.9 \% \mathrm{NaCl}$. The viability of the cells is determined by Trypan blue dye exclusion test and over $95 \%$. Cell count is made using a hematocytometer. In cell count, a completely random distribution of fibrosarcoma Sa-37 tumor cells is observed without the presence of cellular clusters in the cellular suspension.

\section{Tumor growth kinetics}

The period of study comprises the time interval from $t=0$ (initial moment of tumor cells inoculation in the mice) up to tumor reaches a volume $\leq 1.5 \mathrm{~cm}^{3}$. Each individual tumor is observed to verify experimentally the minimum observable tumor volume, named $\mathrm{V}_{\text {obs }}\left(\mathrm{V}_{\text {obs }}<\mathrm{V}_{\text {meas }}\right)$, reached at a time given, $t_{o b s}[2]$. $V_{\text {obs }}$ is observable but not measured. The volume of each individual tumor is calculated by means of the ellipsoid equation $\mathrm{V}=\mathrm{L}_{1} \mathrm{~L}_{2} \mathrm{~L}_{3} / 6 . \mathrm{L}_{1}$, $\mathrm{L}_{2}$ and $\mathrm{L}_{3}\left(\mathrm{~L}_{1}>\mathrm{L}_{2}>\mathrm{L}_{3}\right)$ are three perpendicular tumor diameters. Measurements of $\mathrm{L}_{1}, \mathrm{~L}_{2}$ and $\mathrm{L}_{3}$ are made from tumor reaches $V_{\text {meas }}$ up to $1.5 \mathrm{~cm}^{3}$. A vernier caliper with clamping screw (Model $530-104$ of $0.05 \mathrm{~mm}$ of precision, Mitutoyo, Japan) is used. Each tumor diameter is measured three times for each individual tumor and then averaged, since its edge is not perfectly regular. This method permits tracking tumor development through the study with no need to slaughter the animals. 
Mean doubling time (DT) is estimated for each individual tumor, once it reaches $V_{\text {meas. }}$ DT is the time required for a solid tumor to reach a twofold increase of its initial volume [17].

\section{Form factor and curvature radius of the tumor}

In order to know how tumor shape changes in time, form factor (FF, a measure of curved surface) and curvature radius $\left(R_{c}\right)$ are calculated in three perpendicular planes XY, XZ and YZ. Expressions to calculate FF and $R_{c}$ are shown in Table 1 . FF and $R_{c}$ are calculated for each observation time. In each plane, $R_{c}$ is calculated in the ellipse vertices (points where ellipse curvature is minimized or maximized), named $R_{c-L 1}, R_{c-L 2}$ and $R_{c-L 3}$ (see details in Table 1). FF and $R_{c}$ may be also calculated via measuring all points of this closed quadric surface. In this case, the measurements of these points are tedious and require long time. $\mathrm{L}_{1}, \mathrm{~L}_{2}, \mathrm{~L}_{3}$ and planes $\mathrm{XY}, \mathrm{XZ}$ and $\mathrm{YZ}$ are schematically depicted in Fig. 1a.

\section{Model fitting}

Equations (5) and (6) are used for the first time on the TGK. Below we describe the followed methodology.

First, the non-normalized experimental data are fitted with the Eqs. (3) and (4) from beginning of TGK $(t=0)$. $\tau$ and $V_{\tau}$ values are directly obtained in a plot of the first derivate of tumor volume versus tumor volume, named $V^{\prime}(t)$ versus $V(t)$ plot [2]. In addition, TGK is fitted with the Eqs. (1) and (2) when the first point of the experimental data is $\mathrm{V}_{\mathrm{obs}}, \mathrm{V}_{\mathrm{oo}}$ (tumor volume reaches its diameter of $2 \mathrm{~mm}$ ) or $\mathrm{V}_{\text {meas }}$, satisfying their specific initial conditions $\mathrm{V}(\mathrm{t}=0)=\mathrm{V}_{\mathrm{obs}}, \mathrm{V}(\mathrm{t}=0)=\mathrm{V}_{\mathrm{oo}}$ or $\mathrm{V}(\mathrm{t}=0)=$ $\mathrm{V}_{\text {meas }}$, respectively. These three initial conditions are valid if the respective co-ordinate origin is located at

Table 1 Factor form and curvature radius by planes for the fibrosarcoma Sa-37 tumor

\begin{tabular}{lllll}
\hline Planes & $\begin{array}{l}\text { Form } \\
\text { factor } \\
\text { (FF) }\end{array}$ & \multicolumn{3}{l}{ Curvature Radius $R_{c}$ (in mm) } \\
\cline { 4 - 5 } & $\pi a b / 2 p_{a b}^{2}$ & $b^{2} / a$ & $R_{c-L 2}$ & $R_{c-L 3}$ \\
\hline$x y$ & $\pi a c / 2 p_{a c}^{2}$ & $c^{2} / a$ & - & - \\
xz & $\pi b c / 2 p_{b c}^{2}$ & - & $c^{2} / b$ & $a^{2} / c$ \\
yz & & & $b^{2} / c$ \\
\hline
\end{tabular}

$a\left(a=L_{1} / 2\right), b\left(b=L_{2} / 2\right)$ and $c\left(c=L_{3} / 2\right)$ are the semi-axes of triaxial (or scalene) ellipsoid tumor on their respective planes. $p_{a b}, p_{a c}$ and $p_{b c}$ are the ellipse perimeters on planes $x y, x z$ and $y z$, respectively. $R_{c-L 1}$ is the curvature radius in the point $A, R_{C-L 2}$ in the point $B$ and $R_{C-L 3}$ in the point $C$, as shown in Fig. 1a. It is important to point that the general expression for ellipse curvature radius on each plane is not given because the points of the closed curve do not experimentally measure

$p_{a b}=\pi(a+b)\left[1+\frac{1}{4}\left(\frac{a-b}{a+b}\right)^{2}+\frac{1}{64}\left(\frac{a-b}{a+b}\right)^{4}+\frac{1}{256}\left(\frac{a-b}{a+b}\right)^{6}\right]$

$p_{a c}=\pi(a+c)\left[1+\frac{1}{4}\left(\frac{a-c}{a+c}\right)^{2}+\frac{1}{64}\left(\frac{a-c}{a+c}\right)^{4}+\frac{1}{256}\left(\frac{a-c}{a+c}\right)^{6}\right]$

$p_{b c}=\pi(b+c)\left[1+\frac{1}{4}\left(\frac{b-c}{b+c}\right)^{2}+\frac{1}{64}\left(\frac{b-c}{b+c}\right)^{4}+\frac{1}{256}\left(\frac{b-c}{b+c}\right)^{6}\right]$ $\left(\mathrm{t}_{\mathrm{obs}}, \mathrm{V}_{\mathrm{obs}}\right),\left(\mathrm{t}_{\mathrm{oo}}, \mathrm{V}_{\mathrm{oo}}\right)$ or $\left(\mathrm{t}_{\text {meas }}, \mathrm{V}_{\text {meas }}\right)$. $\mathrm{V}_{\text {obs }}$ and $\mathrm{V}_{\mathrm{oo}}$ are estimated from interpolation and extrapolation methods [2]. These analysis are shown in a $V(t)$ versus $t$ plot to compare the Eqs. (3) and (4), and the Eqs. (1) and (2), and also to know the values and estimation accuracies (or parameter error) of their parameters.

Second, as Eqs. (5) and (6) are normalized between 0 and 1 , the experimental data is normalized by means of the normalization criterion $\mathrm{p}(\mathrm{t})=\left(\mathrm{V}(\mathrm{t})-\mathrm{V}_{\mathrm{i}}\right) /\left(\mathrm{V}_{\mathrm{f}}-\mathrm{V}_{\mathrm{i}}\right) . \mathrm{V}_{\mathrm{i}}$ means the volume fraction of solid tumor at beginning of TGK or when the first point of TGK is $\mathrm{V}_{\mathrm{obs}}, \mathrm{V}_{\mathrm{oo}}$ or $\mathrm{V}_{\text {meas }}$. $\mathrm{V}_{\mathrm{f}}$ represents the volume fraction of the solid tumor at the end of tumor growth. As $V_{i}$ is very small $\left(V_{i}\right.$ tends to 0$)$ this results in $\mathrm{p}(\mathrm{t})=\mathrm{V}(\mathrm{t}) / \mathrm{V}_{\mathrm{f}}$. Normalized experimental data are fitted with the Eqs. (1, 2, 3, 4, 5 and 6), in order to know the parameter values and their estimation accuracies for each equation, as well as to establish a comparison between them.

Third, different graphical strategies are followed, as: $V(t-t)$ versus $t$ (for $t \geq 0)$; $V(t)$ versus $t$ (for $t \geq t_{o b s}$ ); $p(t)$ versus $t$ (for $t \geq 0$ and $\left.t \geq t_{\text {obs }}\right) ; \ln (-\ln (1-p(t)))$ versus $\ln (t)$ on a double-logarithmic plot obtained with the Eq. (5) (for $\mathrm{t}>0) ; \ln \left(-\ln \left(\left(1-\mathrm{p}(\mathrm{t})^{-(\lambda-1)}-1\right) /(\lambda-1)\right)\right)$ versus $\ln (\mathrm{t})$ on a double-logarithmic plot obtained with the Eq. (6) (for $t>0) ; n_{\text {loc }}$ versus $\ln (t)$ and $n_{\text {loc }}$ versus $p(t)$ for both Eqs. (5) and (6), and $t>0 . n_{\text {loc }}\left(n_{\text {loc }} \geq 0\right)$ represents the instantaneous slope of these two equations at any given $p(t)$. All these simulations are made from the mean values of $n, \lambda, K$ and $E_{a}$ obtained from fitting of normalized experimental data with the Eqs. (5) and (6). For the Eq. (5), $\mathrm{n}_{\text {loc }}$ is computed by means of $\partial \ln (1-$ $\mathrm{p}(\mathrm{t})) / \partial \mathrm{t}$. For the Eq. (6), $\mathrm{n}_{\text {loc }}$ is calculated by means of $\partial \ln \left(\left(1-\mathrm{p}(\mathrm{t})^{-(\lambda-1)}-1\right) /(\lambda-1)\right) / \partial \mathrm{t}$. These graphical strategies are suggested by Wang et al. [26].

Fourth, $\mathrm{n}_{\text {loc }}$ is also estimated from the normalized experimental data, for KJMA and mKJMA models. For this, the definition of $\mathrm{n}_{\mathrm{loc}}$, for each model, is applied to the normalized experimental data $(\mathrm{p}(\mathrm{t})$ versus $\mathrm{t}$ plot) when the first point of the experimental data is $V_{\text {obs }}$.

The results of these last three points permit to know if the Eqs. (5) and (6) can be indistinctly used to describe TGK and to give a possible biophysics interpretation of their kinetic parameters.

\section{Criteria for model assessment}

Since tumor growth is represented in biological research as series of volumetric measurements over time, we are presented with a classic case of least squares curve fitting. To fit an $\mathrm{n}$-parameter nonlinear equation to tumor volume measurements, the Marquardt-Levenberg algorithm (an alternative to the Gauss-Newton algorithm) [14, 32] is used, which is the most widely used in nonlinear least squares fitting. Other algorithms have been used, as Nelder-Mead [33], which is not used because the standard deviation of the experimental data is small, even for a larger tumor. 

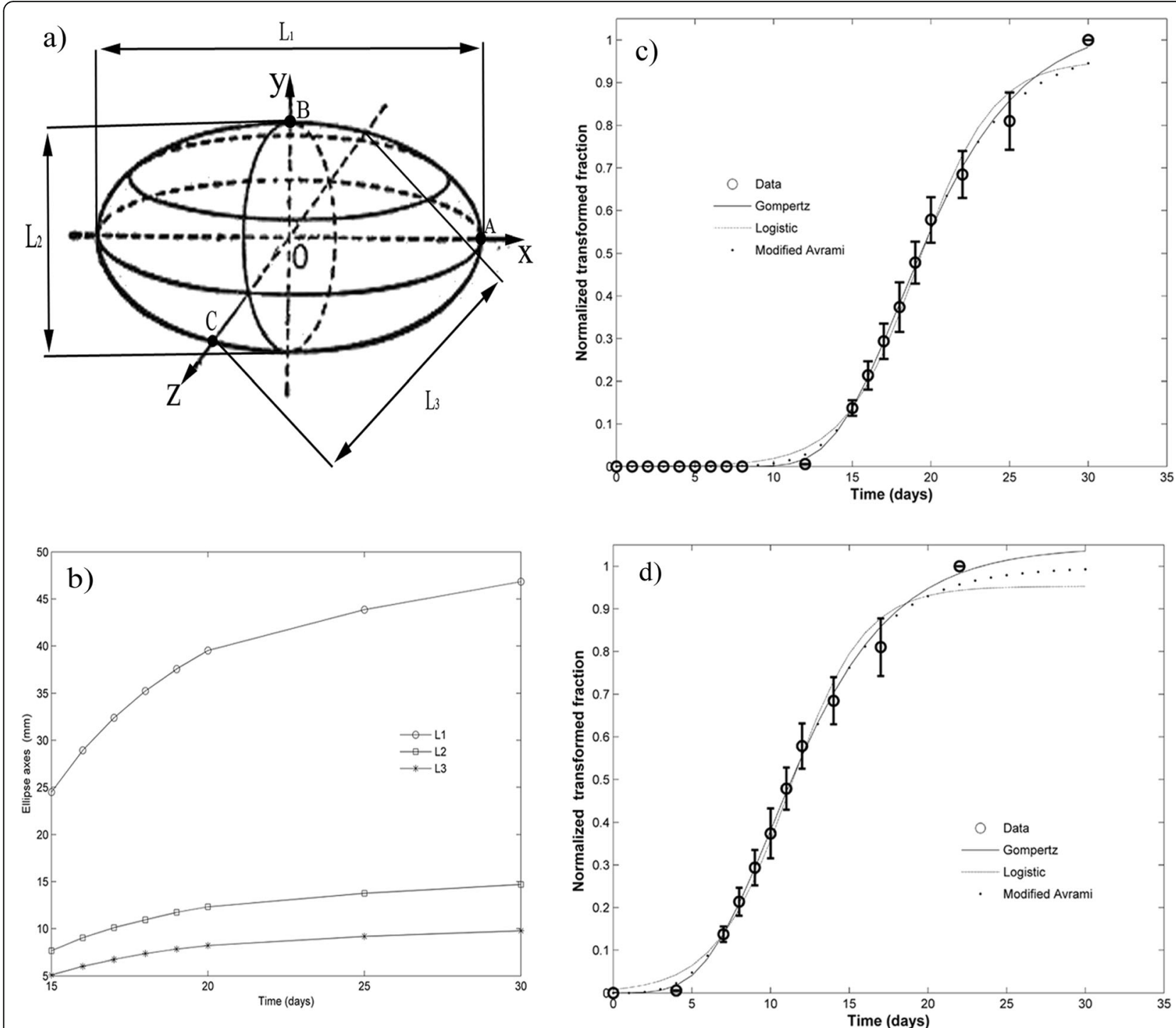

Fig. 1 Fibrosarcoma Sa-37 tumor. a Schematic representation of its triaxial ellipsoid shape of $L_{1}, L_{2}$ and $L_{3}$ diameters. b Time dependences of $L_{1}$, $L_{2}$ and $L_{3}$. Experimental data (Mean \pm standard error) of fibrosarcoma Sa-37 tumor normalized transformed fraction and growth curves fitted with modified models of Gompertz, Logistic and Kolmogorov-Johnson-Mehl-Avrami, from (c) $t=0$ days and (d) $t=8$ days

As the Eqs. (3), (4) and (6) are overparameterized, the parameter estimation accuracy is also obtained from this algorithm. Also, as these three equations are multiparametric and the experimental data have associated error bars, it is important to point out that the error on the fit parameter is calculated multiplying the reported error on the fit parameters by the square root of the reduced chi-squared. For both non-normalized and normalized experimental data, the values and their estimation accuracies of the parameters for Eqs. (1, 2, 3, 4, 5 and 6), and five different fitting quality criteria: the sum of squares of errors, SSE (Eq. 8); standard error of the estimate, SE (Eq. 9); adjusted goodness-of-fit coefficient of multiple determination, $r_{a}^{2}$ (Eq. 10) predicted residual error sum of squares, PRESS (Eq. 11); and multiple predicted residual sum error of squares, MPRESS (Eq. 12) are computed from their individual values and used for model assessment (see details in [4]). These criteria are given by

$$
\begin{aligned}
& S S E=\sum_{j=1}^{n_{1}}\left(\hat{V}_{j}^{*}-V_{j}^{*}\right)^{2}, \\
& S E=\sqrt{\frac{\sum_{j=1}^{n_{1}}\left(\hat{V}_{j}^{*}-V_{j}^{*}\right)^{2}}{n_{1}-k}}, \\
& r_{a}^{2}=1-\frac{n_{1}-1}{n_{1}-k}\left(1-r^{2}\right)=\frac{\left(n_{1}-1\right) r^{2}-k+1}{n_{1}-k}
\end{aligned}
$$




$$
\begin{gathered}
1-r^{2}=\frac{\sum_{j=1}^{n_{1}}\left(\hat{V}_{j}^{*}-V_{j}^{*}\right)^{2}}{\sum_{j=1}^{n_{1}}\left(V_{j}^{*}\right)^{2}-\frac{1}{n_{1}}\left(\sum_{j=1}^{n_{1}} V_{j}^{*}\right)^{2}}, \\
\text { PRESS }=\frac{\sum_{j=1}^{n_{1}-1}\left[\left(\hat{V}_{j}^{*}\right)^{\prime}-V_{j}^{*}\right]^{2}}{n_{1}-k}, \\
\operatorname{MPRESS}(m)=\frac{\sum_{j=m+1}^{n_{1}}\left[\left(\hat{V}_{j}^{*}\right)^{\prime}-V_{j}^{*}\right]^{2}}{n_{1}-m}
\end{gathered}
$$

where $V_{j}^{\prime \prime}$ is the $j$-th measured tumor volume at discrete time $t_{j}, \mathrm{j}=1,2, \ldots, \mathrm{n}_{1}, \hat{V}_{j}^{*}$ is the $j$-th estimated tumor volume by Gompertz, Logistic, KJMA or mKJMA model. $n_{1}$ is the number of experimental points $\left(\mathrm{n}_{1}=11\right) . k$ is the number of parameters. $r^{2}$ and $r_{a}^{2}$ are goodness-of-fit and adjusted goodness-of-fit, respectively. The fitting is considered to be satisfactory when $r_{a}^{2}>0.98$. Higher $r_{a}^{2}$ means a better fit. $\left(V_{j}^{*}\right)^{\prime}$ is the estimated value of $V_{j}^{\prime \prime}$ when the model (Gompertz, Logistic, KJMA or mKJMA model) is obtained without the $j$-th observation. MPRESS removes the last $n_{1}-m$ measurements. The model is fitted to the first $\mathrm{m}$ measured experimental points $(\mathrm{m}=3,4$ or 5$)$ and then from calculated model parameters the error between tumor volume estimates and measured values in the remaining $n_{1}-m$ points is calculated. Least Sum of Squares of Errors is obtained when SSE is minimized in the Marquardt-Levenberg optimization algorithm.

\section{Comparisons between equations}

The Eqs. (3) and (4) are compared when TGK begins at $\mathrm{t}=$ 0 days, taking as reference the Eq. (4). The Eqs. (1) and (2), and the Eqs. (2) and (4) are also compared when the first point of TGK is $\mathrm{V}_{\mathrm{o}}\left(\mathrm{V}_{\mathrm{obs}}, \mathrm{V}_{\mathrm{oo}}\right.$ or $\left.\mathrm{V}_{\text {meas }}\right)$, being the Eq. (2) the reference. Furthermore, the Eqs. (5) and (6) are also compared when the first point of TGK is $\mathrm{V}_{\mathrm{o}}$, using the Eq. (5) as reference. They are also compared with the Eq. (2) (when the first point of TGK is $V_{o}$ ) or the Eq. (4) (when TGK begins at $\mathrm{t}=0$ ). Root Means Squares Errors (RMSE) and maximum distance $\left(D_{\max }\right)$ values are used to compare these equations $[2,14]$, given by

$$
\begin{aligned}
& D_{\max }=\max \left|F_{i}-G_{i}\right| \\
& R M S E=\sqrt{\sum_{i=1}^{M} \frac{\left(F_{i}-G_{i}\right)^{2}}{M}}
\end{aligned}
$$

where $M$ is the total number of points. $G_{i}$ is the $i-t h$ calculated tumor volume with equation choice as reference (see above). $F_{i}$ is the $i$-th calculated tumor volume by another equation compared.

A computer program is implemented in the MATLAB software (version R2011a, license number: 625596, San Jorge University, Zaragoza, Spain) to calculate the values of tumor volume, first derivate of tumor volume, and transformed fraction of tumor volume in each time. In addition, DT; FF; $R_{c}$; RMSE; $\mathrm{D}_{\max }$; SSE; SE; $r_{a}^{2}$; PRESS and MPRESS expressions are implemented in this program to calculate their values.

Each fit with the Eqs. (1, 2, 3, 4, 5 and 6) is performed for each animal's growth curve, for both non-normalized and normalized data. The mean \pm mean standard error of the parameters $L_{1}, L_{2}, L_{3}$, tumor volume, first derivate of the tumor volume, $r^{\prime \prime}, K^{\prime \prime}, \alpha, \beta, F F, R_{c}, \tau, V_{\tau}, K, n, \lambda, E_{a}$, DT, estimation accuracy, RMSE, $D_{\max }, \mathrm{SSE}, \mathrm{SE}, r_{a}^{2}$, PRESS and MPRESS are calculated from their individual values. Mean standard error is calculated as (standard deviation) $/ \sqrt{N}$, where $\mathrm{N}$ is the total number of determinations. $N=3$ is used for each average tumor diameter and $N=28$ for the other parameters. Besides, this software permits performing curve fitting and to visualize the graphs of the graphical strategies above mentioned.

\section{Results}

\section{Unperturbed fibrosarcoma Sa-37 tumor growth kinetics}

The fibrosarcoma Sa-37 tumor exhibits a sigmoidal kinetics characteristic for both non-normalized and normalized experimental data. This $\mathrm{S}$ shape is observed when TGK begins at $\mathrm{t}=0$ (Fig. 1c) or the first point of TGK is $\mathrm{V}_{\mathrm{obs}}$ (Fig. 1d) up to $1.5 \mathrm{~cm}^{3}$, which is reached at 30 days after tumor cells are transplanted into BALB/c mice. Vobs is observed in all tumors between 6 and 9 days. The higher relative frequency of $\mathrm{V}_{\mathrm{obs}}$ is at $\mathrm{t}_{\mathrm{obs}}=8$ days $(24 /$ $28=85.7 \%$ ). The Eq. (4) estimates $V_{\text {obs }}$ in $0.000016 \mathrm{~cm}^{3}$ $(0.031 \mathrm{~cm}$ in diameter $)$ for $t_{\mathrm{obs}}=8$ days. This equation estimates $V_{\text {oo }}\left(0.00416 \mathrm{~cm}^{3}\right)$ at 9.8 days, in agreement with the experiment (around 10 days). $V_{\text {meas }}\left(0.02 \mathrm{~cm}^{3}\right)$ is observed between 10 and 12 days. The higher relative frequency of $V_{\text {meas }}$ is at $t_{\text {meas }}=11$ days $(21 / 28=75 \%)$. The Eq. (4) estimates $V_{\text {meas }}$ at $t_{\text {meas }}=10.8$ days. From $V_{\text {meas }}$, average DT estimated from non-normalized experimental data is $1.6 \pm 0.4$ days.

From $\mathrm{V}_{\text {obs }}$, both tumor and body temperatures remain practically unalterable $\left(36.5 \pm 0.1^{\circ} \mathrm{C}\right)$ for each mouse. As tumor temperature is $36.5^{\circ} \mathrm{C}\left(309.5^{\circ} \mathrm{K}\right)$ and $\mathrm{R}=8.3144 \mathrm{~J} /$ $\mathrm{mol}^{\circ} \mathrm{K}, \mathrm{RT}=2568.85 \mathrm{~J} / \mathrm{mol}$. Besides, surface roughening, compactness and stiffness of the fibrosarcoma Sa-37 tumor increase over time as its volume also increases, verified by both palpation and clinical observation.

Average values of $L_{1}, L_{2}$ and $L_{3}$ values versus time are shown in Fig. 1b, corroborating that the tumor growth is anisotropic (prevails one preferential direction of growth, 
major diameter, named $\left.\mathrm{L}_{1}\right)$. In each mouse, shape changes of fibrosarcoma Sa-37 tumor are observed during entire TGK. Fibrosarcoma Sa-37 tumor grows spherically $\left(\mathrm{L}_{1} \cong\right.$ $\mathrm{L}_{2} \cong \mathrm{L}_{3}$ ) between 6 and 10 days after tumor cells are inoculated in BALB/c mice; then ellipsoidal with slightly irregular border and three different orthogonal well-defined axes $\left(\mathrm{L}_{1}>\mathrm{L}_{2}>\mathrm{L}_{3}\right.$, from 11 up to 17 days); and lastly irregular-shaped, but three diameters of the tumor are still defined and measurable (from 18 up to 30 days). Seventeen days is the time that lapses so that solid tumor reaches $1 \mathrm{~cm}^{3}$. Complete loss of the fibrosarcoma Sa-37 tumor ellipsoidal shape (three diameters of the tumor are not well defined) starts from 30 days post-inoculation, as observed. This and ethical reasons [6] justify why the study period is up to 30 days.

The values of $\tau(15 \pm 2$ days $)$ and $V_{\tau}\left(0.5 \pm 0.05 \mathrm{~cm}^{3}\right)$ are obtained from $V^{\prime}(t)$ versus $V(t)$ plot (results not shown). The higher relative frequency of (15 days, $\left.0.5 \mathrm{~cm}^{3}\right)$ is observed for $57.1 \%(16 / 28)$ of tumors. As a result, in a first approximation, $\tau=15$ days and $V_{\tau}=$ $0.5 \mathrm{~cm}^{3}$ are introduced in the Eqs. (3) and (4) for the simulations.

\section{Parameters of each equation}

Equations (1, 2, 3, 4, 5 and 6) fit well normalized data in each mouse and provided average values of their kinetic parameters, when TGK begins at $\mathrm{t}=0$ (Table 2 and Fig. 1c) or its first point is $V_{\text {obs }}$ (Table 3 and Fig. 1d). For these equations, there is no problem with the convergence in the fitting of individual tumor growth data when the Marquardt-Levenberg optimization algorithm is used. This convergence is rapidly reached. The results are only shown for $\mathrm{V}_{\mathrm{o}}=\mathrm{V}_{\mathrm{obs}}$ in order to know in depth the biggest part of Lag-phase. Comparisons of the Eqs. (1, 2, 3, 4, 5 and 6) are in agreement with small values of SE, SSE, PRESS and MPRESS (Tables 2 and 3), RMSE $\left(\leq 0.001 \mathrm{~cm}^{3}\right)$ and $D_{\max }$ $\left(\leq 0.03 \mathrm{~cm}^{3}\right)$. The mean value \pm mean standard error of $\alpha$, $\beta, r^{*}, K^{\prime \prime}, K, K_{o}, n, \lambda$ and $E_{a}$ parameters and the statistical criteria are given in Tables 2 and 3. The estimation accuracy of the parameters $\alpha, \beta, \mathrm{K}^{\prime \prime}, \mathrm{r}^{\prime \prime}, \mathrm{K}, \mathrm{n}$ and $\lambda$ shown in Table 2 are $0.025 \pm 0.001$ days $^{-1}, 0.015 \pm 0.001$ days $^{-1}, 0.051$ \pm 0.002 days $^{-1}, 0.026 \pm 0.002$ days $^{-1}, 0.002 \pm 0.001$ days $^{-1}$, $0.116 \pm 0.056$ and $0.577 \pm 0.041$, respectively. The estimation accuracy for these respective parameters shown in Table 3 are $0.030 \pm 0.002$ days $^{-1}, 0.021 \pm 0.002$ days $^{-1}, 0.070$ \pm 0.005 days $^{-1}, 0.030 \pm 0.004$ days $^{-1}, 0.008 \pm 0.002$ days $^{-1}$, $0.481 \pm 0.022$ and $0.444 \pm 0.014$.

Although the results of the fitting of the experimental data with Eq. (5) are not shown in Tables 2 and 3, it can be verified that $\mathrm{K}=0.0758$ days $^{-1}$ and $\mathrm{n}=2.7503$. Estimation accuracies of $K$ and $n$ are $0.004 \pm 0.002$ days $^{-1}$ and $0.321 \pm 0.087$, respectively. On the other hand, average DT of $1.7 \pm 0.2$ days is obtained with Eq. (2). Average DT $=0.9 \pm 0.3$ days is predicted with Eq. (6). As expected, these DT values are indistinctly obtained from non-normalized and normalized data.

Table 2 Mean \pm mean standard error of the parameters and criteria for model assessment using in fitting of fibrosarcoma Sa-37 tumor growth data with modified models of Gompertz (Eq. 3), Logistic (Eq. 4) and Kolmogorov-Johnson-Mehl-Avrami (mKJMA) (Eq. 6) from $t \geq 0$ days

\begin{tabular}{|c|c|c|c|}
\hline & \multicolumn{3}{|c|}{ Modified models on normalized data } \\
\hline & Gompertz & Logistic & mKJMA \\
\hline $\mathrm{X}_{1} \pm S \mathrm{SD}$ & $(0.473 \pm 0.053)$ days $^{-1}$ & $(0.953 \pm 0.031) \mathrm{cm}^{3}$ & $(0.052 \pm 0.003)$ days $^{-1}$ \\
\hline$X_{2} \pm S D$ & $(0.233 \pm 0.031)$ days $^{-1}$ & $(0.425 \pm 0.043)$ days $^{-1}$ & $7.599 \pm 1.247$ \\
\hline$X_{3} \pm S D$ & - & - & $2.271 \pm 0.521$ \\
\hline$r^{2} \pm S D$ & $0.995 \pm 0.004$ & $0.989 \pm 0.007$ & $0.994 \pm 0.004$ \\
\hline$r_{a}^{2} \pm S D$ & $0.995 \pm 0.004$ & $0.989 \pm 0.007$ & $0.994 \pm 0.004$ \\
\hline $\mathrm{SE} \pm \mathrm{SD}$ & $0.022 \pm 0.007$ & $0.032 \pm 0.009$ & $0.023 \pm 0.007$ \\
\hline$S S E \pm S D$ & $0.009 \pm 0.006$ & $0.019 \pm 0.0115$ & $0.010 \pm 0.006$ \\
\hline $\mathrm{PRESS} \pm \mathrm{SD}$ & $0.0004 \pm 0.0003$ & $0.0008 \pm 0.0004$ & $0.0004 \pm 0.0002$ \\
\hline MPRESS1 \pm SD & $0.0006 \pm 0.0004$ & $0.0012 \pm 0.0007$ & $0.0006 \pm 0.0004$ \\
\hline MPRESS2 $\pm S D$ & $0.0006 \pm 0.0004$ & $0.0013 \pm 0.0007$ & $0.0007 \pm 0.0004$ \\
\hline MPRESS3 \pm SD & $0.0007 \pm 0.0004$ & $0.0014 \pm 0.0007$ & $0.0007 \pm 0.0004$ \\
\hline $\mathrm{K}_{\mathrm{o}}\left(\right.$ days $\left.^{-1}\right)$ & - & - & $0.056 \pm 0.004$ \\
\hline $\mathrm{E}_{\mathrm{a}}(\mathrm{J} / \mathrm{mol})$ & - & - & $187.331 \pm 157.609$ \\
\hline $\mathrm{RT}(\mathrm{J} / \mathrm{mol})$ & - & - & 2568.85 \\
\hline
\end{tabular}

$\mathrm{X}_{1}$ and $X_{2}$ variables signify the parameters $\alpha$ and $\beta$ in the modified Gompertz model whereas these two variables symbolize the parameters $\mathrm{K}^{*}$ and $\mathrm{r}^{*}$ in the modified Logistic model, respectively. $X_{1} X_{2}$ and $X_{3}$ represent $K, n$ and $\lambda$ in mKJMA model, respectively. RT is the thermal energy calculated. T, $K_{0}, E_{a}, S E$, SSE, $r_{a r}^{2}$ PRESS, MPRESS and SD are the temperature, pre-exponential factor, activation energy (activation enthalpy) of the transformation, standard error of the estimate, sum of squares of errors, adjusted $r^{2}$, predicted residual error sum of squares, multiple predicted residual sum error of squares and standard deviation respectively. $r^{2}$ is the goodness-of-fit. Details of SE, SSE, $r^{2}, r_{a}^{2}$ PRESS and MPRESS are given in $[2,14]$ 
Table 3 Mean \pm mean standard error of the parameters and criteria for model assessment using in fitting of fibrosarcoma Sa-37 tumor growth data with modified models of Gompertz (Eq. 2), Logistic (Eq. 1) and Kolmogorov-Johnson-Mehl-Avrami (mKJMA) (Eq. 6) from $t \geq 8$ days

\begin{tabular}{|c|c|c|c|}
\hline & \multicolumn{3}{|c|}{ Modified equations on normalized data } \\
\hline & Gompertz & Logistic & mKJMA \\
\hline $\mathrm{X}_{1} \pm \mathrm{SD}$ & $(0.473 \pm 0.053)$ days $^{-1}$ & $0.953 \pm 0.031$ & $(0.083 \pm 0.008)$ days $^{-1}$ \\
\hline$X_{2} \pm S D$ & $(0.233 \pm 0.031)$ days $^{-1}$ & $(0.425 \pm 0.043)$ days $^{-1}$ & $3.409 \pm 0.529$ \\
\hline $\mathrm{X}_{3} \pm \mathrm{SD}$ & - & - & $1.509 \pm 0.366$ \\
\hline$r^{2} \pm S D$ & $0.991 \pm 0.005$ & $0.982 \pm 0.011$ & $0.991 \pm 0.006$ \\
\hline$r_{a}^{2} \pm S D$ & $0.990 \pm 0.007$ & $0.979 \pm 0.013$ & $0.990 \pm 0.007$ \\
\hline $\mathrm{SE} \pm \mathrm{SD}$ & $0.030 \pm 0.009$ & $0.044 \pm 0.013$ & $0.030 \pm 0.009$ \\
\hline $\mathrm{SSE} \pm \mathrm{SD}$ & $0.009 \pm 0.006$ & $0.019 \pm 0.010$ & $0.009 \pm 0.006$ \\
\hline $\mathrm{PRESS} \pm \mathrm{SD}$ & $0.0008 \pm 0.0005$ & $0.0015 \pm 0.0008$ & $0.0007 \pm 0.0005$ \\
\hline MPRESS $1 \pm S D$ & $0.0011 \pm 0.0007$ & $0.0022 \pm 0.0014$ & $0.0010 \pm 0.0007$ \\
\hline MPRESS2 \pm SD & $0.0012 \pm 0.0008$ & $0.0024 \pm 0.0016$ & $0.0012 \pm 0.0007$ \\
\hline MPRESS3 \pm SD & $0.0014 \pm 0.0009$ & $0.0026 \pm 0.0018$ & $0.0013 \pm 0.0009$ \\
\hline $\mathrm{K}_{0}\left(\right.$ days $\left.^{-1}\right)$ & - & - & $0.109 \pm 0.014$ \\
\hline $\mathrm{E}_{\mathrm{a}}(\mathrm{J} / \mathrm{mol})$ & - & - & $706.97 \pm 393.15$ \\
\hline RT (J/mol) & - & - & 2568.85 \\
\hline
\end{tabular}

$\mathrm{X}_{1}$ and $X_{2}$ variables signify the parameters $\mathrm{a}$ and $\beta$ in the modified Gompertz equation whereas these two variables symbolize the parameters $\mathrm{K}^{*}$ and $\mathrm{r}^{*}$ in the modified Logistic equation, respectively. $X_{1} X_{2}$ and $X_{3}$ represent $K, n$ and $\lambda$ in Modified KJMA equation, respectively. RT is the thermal energy calculated. SE: Standard error of the estimate. SSE sum of squares of errors. $r_{a}^{2}$ : adjusted $r^{2}$. PRESS Predicted residual error sum of squares and MPRESS Multiple predicted residual sum error of squares. SD Standard deviation. $\mathrm{K}_{\mathrm{o}}$ is the pre-exponential factor. $\mathrm{E}_{\mathrm{a}}$ is the activation energy (activation enthalpy) of tumor cell nucleation. $r^{2}$ is the goodness-of-fit. Details of SE, SSE, $r^{2}, r_{a}^{2}$, PRESS and MPRESS are given in $[2,14]$

Tables 2 and 3 show that parameters $\alpha, \beta, K^{\prime \prime}$ and $r^{*}$ have equal values. $\alpha$ and $\beta$ values differ from those reported by Cabrales et al. [2] in 0.04 and 0.033 days $^{-1}$, respectively. Values for $\alpha$ and $\mathrm{r}^{*}$ differ in 0.048 days $^{-1}$, indicating that $\alpha \cong r^{\prime \prime}$. In addition, Tables 2 and 3 evidence that $K$ values are one order smaller than $\alpha$ and $r^{*}$ values, and the values of $E_{a}$ are smaller than $R T . K_{o}, K$ and $E_{a}$ values shown in the Table 3 are higher than those in Table 2. Values for $\mathrm{n}$ and $\lambda$ shown in Table 3 are smaller than those in the Table 2. Although the results are not shown, it can be verified that $K_{o}, K$ and $E_{a}$ values increase, and $n$ and $\lambda$ values decrease with respect to those shown in Table 3 when tumor volume increases regarding to $V_{\text {obs }}$.

On the other hand, it can be verified that results shown in Table 3 coincide with those obtained from fitting of no-normalized data with Eqs. (1, 2, 3 and 4), when the first point of experimental data is $\mathrm{V}_{\mathrm{obs}}, \mathrm{V}_{\mathrm{oo}}$ or $\mathrm{V}_{\text {meas }}$. Nevertheless, when TGK begins for a tumor volume higher than $V_{\text {meas }}, \alpha, \beta, \mathrm{K}^{*}$ and $\mathrm{r}^{*}$ change compared with those shown in Table 3 (results not shown). In addition, Eqs. (3) and (4), and Eqs. (1) and (2) fit well to no-normalized data in each mouse when TGK beginning at $\mathrm{t}=0$ and the first point is $\mathrm{V}_{\mathrm{obs}}, \mathrm{V}_{\mathrm{oo}}$ or $\mathrm{V}_{\text {meas }}$.

Figure 2 shows that $F F$ and $R_{c}$ depend on time and the plane $\mathrm{XY}, \mathrm{XZ}$ or $\mathrm{YZ}$. The higher values of $\mathrm{FF}$ and $\mathrm{R}_{\mathrm{c}}$ are observed in plane $Y Z$ and $L_{1}$ diameter (along axis $x$ ), respectively. Moreover, this figure reveals that $R_{c-L 1}, R_{c-L 2}$ and $R_{c-L 3}$ increase with time, being $R_{c-L 1}>R_{c-L 2}>R_{c-L 3}$.
The graphical strategies for constant temperature show similar behaviors to those shown in [26] and therefore, they are not shown in this study. Nevertheless, it can be verified that simulations of $\ln (-\ln (1-\mathrm{p}(\mathrm{t})))$ versus $\ln (\mathrm{t})$ plot and $\ln \left(-\ln \left(\left(1-\mathrm{p}(\mathrm{t})^{-(\lambda-1)}-1\right) /\right.\right.$ $(\lambda-1)))$ plot exhibit linear and non-linear increases, respectively. $\mathrm{n}_{\text {loc }}$ versus $\mathrm{p}(\mathrm{t})$ plot shows that $\mathrm{n}_{\text {loc }}$ remain constant for KJMA model, whereas $n_{\text {loc }}$ non-linearly decreases as $p(t)$ increases, for mKJMA model. This non-linearity is noticeable when $\lambda$ increases. In addition, simulation of $\mathrm{n}_{\mathrm{loc}}$ versus $\ln (\mathrm{t})$ plot for Eq. (5) predicts a linear behavior of $\mathrm{n}_{\mathrm{loc}}$ in the time. However, this plot for Eq. (6) evidences that $\mathrm{n}_{\text {loc }}$ drops exponentially in the time (continue and smooth curve). This deviation from linearity starts at the very early stages of the entire TGK, when $\lambda>1$, being noticeable when $\lambda$ increases.

The analysis of $n_{\text {loc }}$ versus $\ln (t)$ plot on the normalized experimental data reveals that $\mathrm{n}_{\mathrm{loc}}$ drops with time showing a jump (around 10 days) for both KJMA and mKJMA models, as it can be seen in Fig. 3 . It is important to point out that this jump coincides with the shift in the fibrosarcoma Sa-37 tumor from spherical to ellipsoidal shape. Obtained values for $\mathrm{n}_{\text {loc }}$ with mKJMA are higher than those for the KJMA model. Besides, for both models, $\mathrm{n}_{\mathrm{loc}}>4$ (before 6 days) and $3 \leq n_{\text {loc }} \leq 4$ (between 6 and 10 days) are observed. 
a)

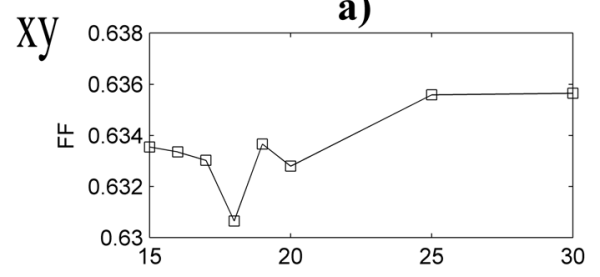

$\mathrm{XZ}$

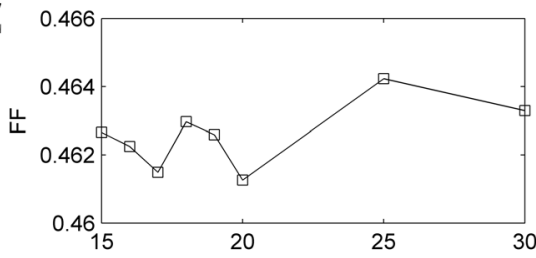

yZ

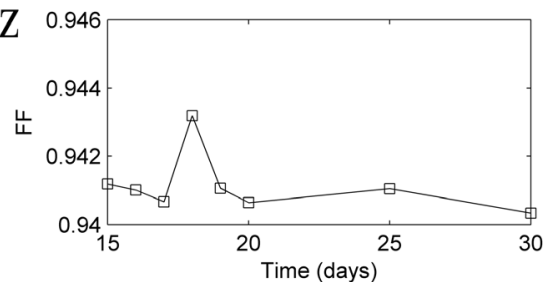

b)
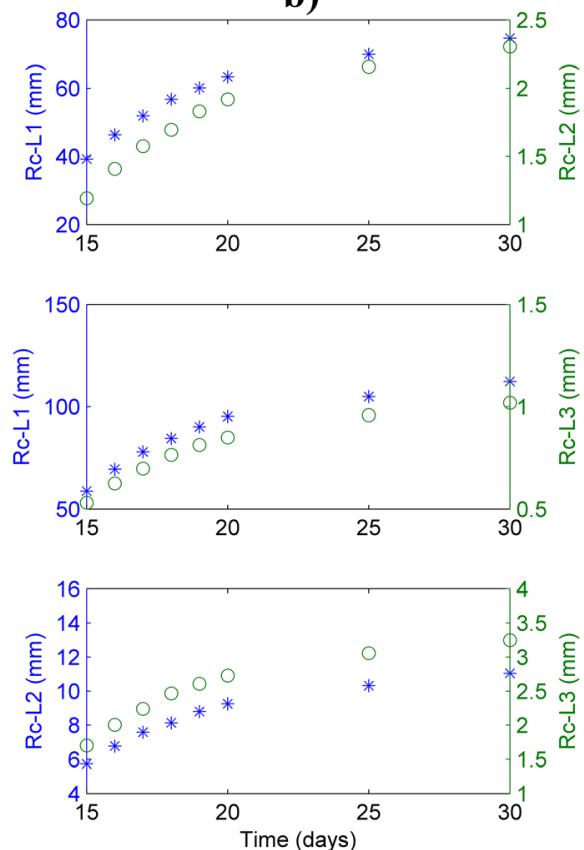

Fig. 2 Shape change of fibrosarcoma Sa-37 tumor. a Tumor factor form (FF) versus time. b Tumor curvature radius versus time on points A, B and C, given by $R_{c-L 1}, R_{c-L 2}$ and $R_{C-L 3}$, respectively. $F F, R_{c-L 1}, R_{c-L 2}$ and $R_{c-L 3}$ are given on three perpendicular planes $x y, x z$ and $y z$. These curves are shown for $t \geq t_{0 b s}$

\section{Discussion}

The results of this study are valid for the unperturbed fibrosarcoma Sa-37 tumor, experimentally transplanted to $\mathrm{BALB} / \mathrm{c}$ mice. As shown, parameter $\mathrm{n}_{\text {loc }}$ is a better descriptor than $\mathrm{n}$ for the entire TGK. The plausibility of $\mathrm{V}(\mathrm{t})$ versus $\mathrm{t}$ plot and/or $\mathrm{p}(\mathrm{t})$ versus $\mathrm{t}$ plot for TGK analysis is also suggested, in agreement with [34]. Equations (1, 2, 3, 4, 5 and 6) can be used to fit normalized experimental data from Sa-37 tumor, as assessed by the high $r_{a}^{2}$ values, low values of SSE, SE, PRESS, MPRESS as well as overall estimation accuracy. Each equation has high prediction capability and good missing data handling. This further supports sigmoid laws universality $[3,35]$.

Despite mentioned in the previous paragraph, a weighted least square minimization in formula (6) may

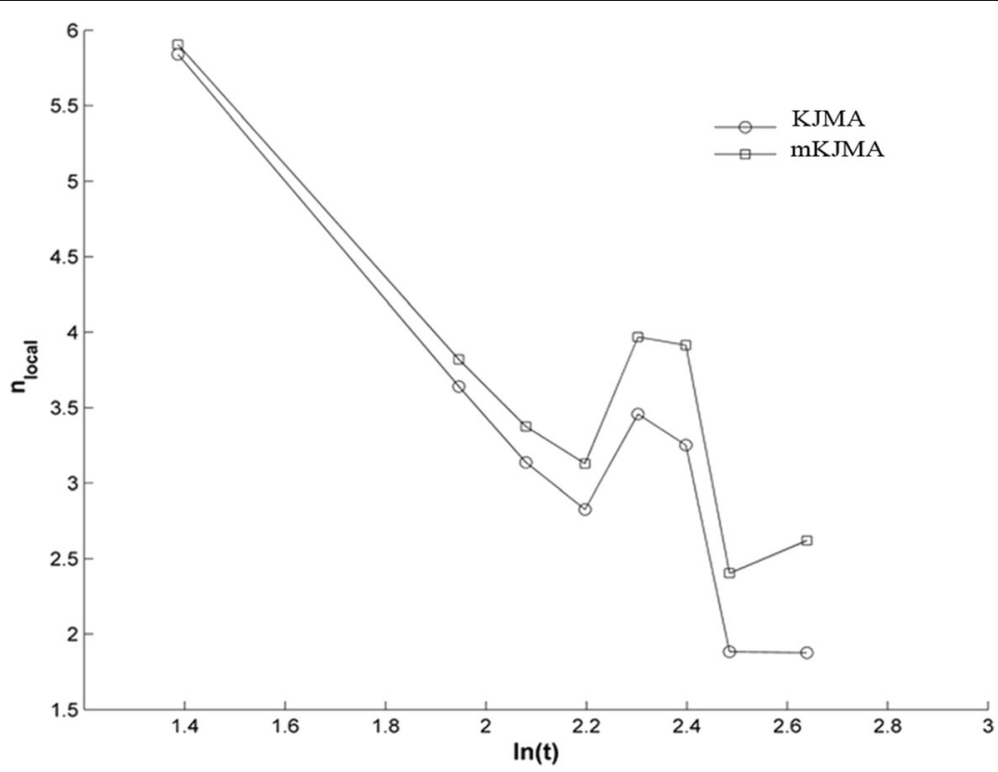

Fig. $3 n_{\text {loc }}$ versus $I n(t)$ plot on the normalized experimental data for KJMA and mKJMA models, and $t \geq t_{\text {obs }}$ 
be proposed for selection of the best model, taking into account the uncertainty of the individual measurements of the tumor volume and the fact that the larger the volume, the larger the standard deviation. This and other statistical criteria [33] in tumor volumes with smaller and larger standard deviations will be included in a further study.

As obtained, $\mathrm{V}_{\mathrm{o}}$ can be indistinctly chosen as $\mathrm{V}_{\mathrm{obs}}, \mathrm{V}_{\mathrm{oo}}$ or $V_{\text {meas }}$ since Eq. (2) behaves similarly when any of them is used in experimental data fitting. Unlike Eqs. (2) and (4), the parameters of Eq. (6) depend on the first point of TGK, indicating that it senses the microstructural changes from beginning of TGK $(t=0)$.

The good fits yielded by Eqs. (1) and (3) are in contrast with $[8,9,11,33]$. This can be due to the omission of larger tumors, since mice were slaughtered earlier, following [6]. That is why, $\mathrm{p}(\mathrm{t})$ and $\mathrm{n}_{\mathrm{loc}}$ do not reach the values of 1 and 0 , respectively. In crystals, $p(t)=1$ and $\mathrm{n}_{\text {loc }}=0[26]$.

Equation (5) should not be used for TGK interpretation, since $\lambda>1$; its parameters differ respect to those of Eq. (6) (Tables 2 and 3, and Fig. 3) and graphical strategies are noticeably different for these two equations. This agrees with Wang et al. [26]. Accordingly, results obtained with Eq. (5) have not been exposed here.

The close relationship between fibrosarcoma Sa-37 tumor spherical shape and $n_{\text {loc }} \geq 3$ is corroborated in this study. Similar finding is reported in crystals [28-30]. This tumor spherical shape may be vital for tumor growth due to a lower surface curvature, in agreement with [2,36-38]. Jump of $\mathrm{n}_{\text {loc }}$ and the change from spherical to non-spherical shape may be related to a shift from avascular (before 10 days) to vascular growth phase (after 11 days). Transition between these two phases has been previously reported [36, 37]. The observed $n_{\text {loc }}$ jump corresponds to a transition of high (before $n_{\text {loc }}$ jump) to low (after $n_{\text {loc }}$ jump) value of $n_{\text {loc }}$, suggesting the occurrence on TGK of two types of growth mechanisms that happen at different time scales: nucleation (below 10 days) and pure growth (above 11 days). Nucleation is expected at vascular growth phase, mainly at its very early stages, by high values of $n_{\text {loc }}$ and it is the stochastic stage of a forming and growing system. This later may be due to the Brownian motion (a fractal stochastic process) of thermally fluctuating and energetically unstable tumor cells in suspension at $\mathrm{t}=0$.

High energetic instabilities at avascular growth phase are mitigated by nucleation mechanisms, suggesting a high micro-anisotropy, confirmed by $n_{\text {loc }} \geq 5$. Microanisotropy leads to random formation of non-uniform and energetically unstable cellular micro-clusters, which establish a space-time competence for nutrients, oxygen and energy, resulting in high micro-heterogeneities, as reported in multicellular spheroid models [36-38]. This may explain the existence of the entropy production [39] and the diffusion limited aggregation at avascular tumor growth (mainly at its very early stages of TGK) because the tumor cells move randomly in Brownian motion, forming fractal clusters when diffusion is the main transport mechanism. Brownian motion and diffusion limited aggregation are stochastic rather than deterministic processes with random fractal dynamics. This diffusion limited aggregation may have an impact in TGK [40] and result in tumor cells packed in a multicellular spheroid not yet connected to the host's blood supply, in agreement with [36-39, 41].

The formation of these cellular micro-clusters discards the occurrence of a burst nucleation, which means that all nucleation sites are immediately saturated at $\mathrm{t}=0$. Burst nucleation is reached for $\mathrm{K} \rightarrow \infty, \lambda=1, \mathrm{n} \rightarrow \infty$ and/or DT $\rightarrow 0$, in contrast with the results of this paper and with duration of Lag stage of TGK observed in preclinical (several days) and in clinical (several months and years) studies. Additionally, the existence of cellular micro-clusters may suggest that a tumor solid seed (or smallest size of a solid tumor), long before of $\mathrm{V}_{\mathrm{obs}}$, may be essentially formed via heterogeneous nucleation mechanisms, as previously hypothesized Cabrales et al. [2]. This via is confirmed in this study by non-integer values of $\mathrm{n}$ and $\mathrm{n}_{\text {loc }}$, as in crystals [28-30].

Nucleation mechanisms may help to form these cellular micro-clusters by filling the high nucleation sites (or vacancies), which may correspond with unoccupied sites of the cancer cells. The existence of these sites may be justified because $n_{l o c} \geq 5$; this can lead to a higher number of heterogeneous sites, making unstable both the forming cellular system and the cellular microclusters. This process may be stabilized and ordered by both inter-cellular interactions and the overlapping of diffusion fields of tumor cells, a matter that agrees with $[19,36,41]$, suggesting the existence of soft impingement mechanisms during the avascular growth phase. These mechanisms are also confirmed because $\lambda>2$, as in crystals [26-28]. Nucleation and soft impingement mechanisms may explain, in part, why a slightly better binding of cancer cells with less detachment, in agreement with [42].

The filling of vacancies may explain why $n_{\text {loc }}$ drops up to the jump of $n_{\text {loc }}$. After $n_{\text {loc }}$ jump, $n_{\text {loc }}$ increases probably because pure growth mechanisms emerge and prevail over nucleation mechanisms. If pure growth mechanisms do not emerge, nucleation sites are completely saturated ( $\mathrm{n}_{\text {loc }}$ tends to 0 ) in less than 30 days, in contrast with the results shown in Fig. 3 . It should be expected that $\mathrm{n}_{\text {loc }}$ tends to 0 for larger tumors $\left(\geq 3 \mathrm{~cm}^{3}\right.$, which is reached long past 30 days) because TGK decelerates at stationary stage of TGK (cell-production-tocell-loss rate is very slow or unalterable). This ratifies that TGK cannot be linear nor exponential (the host 
cannot fully sustain solid tumors due to their sizes would be bigger than host size). Accordingly, solid tumors are cooperative boundless systems, in agreement with the S-shape of tumor growth, and the fact that Eq. (6) has to level off at both extremes to represent almost no binding at the beginning of TGK and saturated binding at the final of TGK.

Heterogeneity and anisotropy of the fibrosarcoma Sa37 tumor at vascular growth phase are confirmed by palpation; time changes of $n_{\text {loc }}, L_{1}, L_{2}, L_{3}, F F$ and $R_{c}$; irregular border, deformation and surface roughening $[2,17,25]$ and are associated with compactness, stiffness and surface tension of the tumor [23, 24, 43]. Anisotropy produces preferred directions of growth, minimizing surface tension.

Brownian motion and cellular micro-clusters at very early stages of TGK; Figs. 1, 2 and 3; and the irregular border, surface roughening and stiffness of the tumor at vascular growth phase may suggest that forming, growing and transforming cellular system along TGK happens in a fractal space-time; as a consequence the fractional Hausdorff dimension $\left(D_{H}\right)$ is higher than the topologic dimension $\left(\mathrm{D}_{\mathrm{T}}\right)$, as it corresponds to a fractal space [44]. This means that although $\mathrm{D}_{\mathrm{T}}=0$ for tumor cells in suspension (considered as a set of points) at $\mathrm{t}=0,0<\mathrm{D}_{\mathrm{H}}<1$. It is expected that the forming and growing cellular system on TGK pass through different spatial patterns, starting from worm-like linear structures $\left(D_{T}=1\right.$ and $\left.1<D_{H}<2\right)$; then, fish-like plane structures $\left(D_{T}=2\right.$ and $\left.2<D_{H}<3\right)$; spatial solid-like structures $\left(D_{T}=3\right.$ and $\left.3<D_{H}<4\right)$; and lastly, space-time structure $\left(D_{\mathrm{T}}=4\right.$ and $4<\mathrm{D}_{\mathrm{H}}<5$ or higher dimensions). It is possible that these two later structure types are reached once the tumor solid seed and vascular growth phase are formed, respectively. This is in contrast with [45] and agrees with [46]. Waliszewski and Konarski [45] obtain that the value of the mean temporal fractal dimension decreases along the curve approaching integer value because the fractal structure is lost with tumor progression. Shim et al. [46] correlate the S-shaped time increase of tumor fractal dimension, with textural parameters (i.e., hardness) and the growth in the time of space-time branching structures (or patterns). These structures are linked to the abnormal network of blood vessels, in agreement with the findings of the present study. This and the inverse relation between $p(t)$ and $\mathrm{n}_{\text {loc }}$ (Figs. 1, 2 and 3) may suggest that $\mathrm{n}_{\text {loc }}$ and the tumor fractal dimension are inversely related, as it takes place in crystals [30]. Time changes in $\mathrm{D}_{\mathrm{T}}$ and $\mathrm{D}_{\mathrm{H}}$ may explain, in part, why tumor cells in vitro form colonies and grow in layers, unlike the normal cells, which do not form colonies [47].

Fractal properties of tumors have been correlated with its microstructure, microscopic coherent local deformation processes (or local dynamical rearrangements), mitosis rate, heterogeneity, anisotropy, complexity degree, spatialtemporal coherence, self-organization, self-stabilization, self-symmetry, self-ordering, self-similarity, mechanical properties (stiffness, compactness and surface roughening), temporal changes of nontrivial shape and dynamical structural intrinsic transformations [21, 35, 48-51].

Tumor fractal dimension may suggest that the tumor is a type of fractal, named space-filling fractal that continuously attempts to fill in the area leaving no empty holes. The space-filling pattern is formed by placing some non-overlapping units of smaller sizes. This may confirm the existence of annihilation of vacancies, in agreement with Molski and Konarski [48], confirming that solid phase of TGK is spatially coherent and therefore, all tumor cells co-operate collectively producing spatial-temporal organization and complex patterns.

The above discussed suggests that TGK is a fractal from its beginning $(t=0)$, unprecedented in the literature. This statement agrees with [52], in which is demonstrated the fractal origin of the Gompertz equation. Izquierdo-Kulich et al. [52] explain their results because $\alpha$ and $\beta$ are connected with morphology of the tumors, specifically with the fractal character of them.

Small values of $E_{a}$ and minimal tumor surface tension [23] may explain time evolution of $\mathrm{V}(\mathrm{t}), \mathrm{p}(\mathrm{t}), \mathrm{n}_{\text {loc }}, \mathrm{D}_{\mathrm{T}} /$ $\mathrm{D}_{\mathrm{H}}$, shapes of the fibrosarcoma Sa-37 tumor. In addition, small values of $E_{a}$ corroborate that vacancies require small amount of energy for their creations. They have received insufficient attention and may have an important role in the carcinogenesis, production/lost rate of tumor cells, and in mechanics $[23,24]$ and dielectric [53] properties of a forming and growing cellular system, during entire TGK. This may be explained because vacancies disorder these cellular systems, leading to structural, morphological and electrical instabilities $[21,48,49,51]$. As a result, impingement mechanisms emerge during TGK in order to guarantee the most efficient space-filling and to stabilize the forming and growing cellular system, in order to maximize its exchanges of nutrients in the minimum amount of space and therefore to maximize the tumor growth and its stabilization. After $\mathrm{n}_{\text {loc }}$ jump, soft impingement mechanisms guarantee the growth, stabilization and survival of the tumor by branching structure (abnormal vascular network) [14-17], the different abnormal signaling pathways, the interactions that happen in the tumor and/or other uncontrolled environmental factors $[17,23,36,54,55]$. As a result, the tumor cells do not multiply in an unregulated manner, as reported in [17], but they are regulated by the number of vacancies available to be filled. Furthermore, intrinsic local dislocations lead to dynamical rearrangements of tumor cells, suggesting that dynamical structural intrinsic 
transformations take place along the entire TGK. This indicates that the forming and growing cellular system passes through different dynamical conformational states or meta-stable configurations. These configurations are rearrangements of the cancer cells that take place over a wide energy range due to the large number of stabilized and ordered cellular configurations. This agrees with Guha [56], who reports that a change of state takes place if there is an unbalanced force anywhere within the system, or between the system and its surrounding, leading to variations in pressure or elastic stress which give rise to the tumor expansion. This brings about that TGK may be limited and controlled by vacancies, which are governed by nucleation/growth and impingement mechanisms, and dynamical structural intrinsic transformations. As a result, cancer selfrenews constantly and TGK is a highly coordinated dynamic multi-step process, in agreement with [57].

On the other hand, these dynamical structural intrinsic transformations may explain, in part, immune resistance mechanisms and low effectiveness of some antitumor therapies (i.e., immunotherapy when solely applied), in agreement with [58]. This may be explained because these transformations may be responsible for structural and stereochemical changes on membrane-bound receptor-ligand immune checkpoints that promote the tumor activity. As a result, ligand-receptor interactions are perturbed due to the expression of immunecheckpoint proteins which are disregulated [58].

The results may evidence that entire TGK is not only due to imbalance between cell production and cell loss [17] and other hallmarks of cancer [17, 54, 55], but also to diffusion-controlled nucleation/growth and impingement mechanisms, and dynamical structural intrinsic transformations, which may be the key to understand how a solid tumor arises and grows. These findings are often ignored in literature and may indicate that TGK is about dynamical structural transformations, instead of pure growth kinetics. They may explain why $\mathrm{K}$ is an order smaller than $\alpha / r^{\prime \prime}$; DT value estimated with the Eq. (6) is smaller than that estimated experimentally and with the Eq. (2); the differences between the values of $\mathrm{K}$, $\mathrm{n}, \lambda$ and $E_{a}$ report in the Tables 2 and 3; and the difference of $n_{\text {loc }}$ versus $\ln (t)$ for KJMA and mKJMA models (Fig. 3). On the other hand, if these findings are not considered on entire TGK, then pure growth mechanisms prevail in it, meaning that $K \cong \alpha \cong r^{*}$ and $D T$ estimated experimentally and with Gompertz, Logistic and mKJMA models are equals, in contrast with results here shown.

Besides, the prevalence of these findings at avascular growth phase ratify that an important part of vital cycle of a solid tumor occur before it is clinically detected, in agreement with [17]. On the other hand, the Eq. (6) senses the microstructural changes that happen during the entire TGK, mainly at avascular growth phase, in contrast to Eqs. (1, 2, 3 and 4).

Many questions may arise, as: how $\mathrm{n}, \mathrm{n}_{\mathrm{loc}}, \lambda$ and $\mathrm{E}_{\mathrm{a}}$ depend on $\alpha, \beta$ and DT, which characterize the histological characteristics of a solid tumor? How an immunedeficient or immune-competent organism affect $n, n_{\text {loc }}, \lambda$ and $E_{a}$ values? Can the Eq. (6) be modified to fit the perturbed tumor growth kinetics with an external agent? among others. This first study cannot give answers to all these questions. Relevant biological and clinical data may now be gathered in a systematic manner in order to test our theory or any other quantitative model derived using a methodology similar to ours, with the aim of helping to understand, and potentially handling, the process of tumor growth. Future studies will provide in-depth experimental findings that permit a best interpretation of the parameters of mKJMA model in cancer.

\section{Conclusions}

In conclusion, modified Kolmogorov-Johnson-MehlAvrami is adequate to describe unperturbed transplanted fibrosarcoma Sa-37 tumor growth, which is not a purely growth kinetics, but kinetics of dynamical structural intrinsic transformation, involving diffusion-controlled nucleation/growth and impingement mechanisms. Besides, TGK follows a fractal nucleation and growth model.

\begin{abstract}
Abbreviations
$\lambda$ : Impingement factor; $a$ : The intrinsic growth rate of the tumor; $\tau$ : The time that corresponds to inflection point of TGK; $r_{a}^{2}$ : Adjusted goodness-of-fit coefficient of multiple determination; $D_{H}$ : Hausdorff dimension;

$D_{\text {max: }}$ : Maximum distance; $\mathrm{D}_{\mathrm{T}}$ : Topologic dimension; DT: Tumor doubling time; $E_{a}$ : Effective (overall) activation energy of the transformation; FF: Tumor form factor; $K^{*}$ : The carrying capacity; K: Specific rate of transformation process; KJMA: Kolmogorov-Johnson-Mehl-Avrami classical model; $K_{0}$ : The pre-exponential factor; mKJMA: Modified Kolmogorov-Johnson-Mehl-Avrami model; MPRESS: Multiple predicted residual sum error of squares; $n$ : The Avrami exponent; $p(t)$ : The transformed tumor fraction at $t$; PRESS: Predicted residual error sum of squares; $r^{*}$ : The growth rate; $R$ : Boltzmann constant; $R_{c}$ : Tumor curvature radius; RMSE: Root Means Squares Errors; RT: Represents the thermic kinetics energy; RT: The thermic kinetics energy; SE: Standard error of the estimate; SSE: The sum of squares of errors; T: Temperature; TGK: Tumor growth kinetics; $V_{T}$ : Tumor volume corresponding to inflection point of TGK; $V(t-\tau)$ : Tumor volume at time $(t-T) ; V_{\text {meas }}$ : Minimum measurable tumor volume; $V_{0}$ : Initial tumor volume; $V_{\text {obs }}$ : Minimum observable tumor volume; $V_{o o}$ : Tumor volume reaches a diameter of $2 \mathrm{~mm}$; $\beta$ : The growth deceleration factor related to the endogenous antiangiogenesis processes
\end{abstract}

\section{Acknowledgements}

All authors are supported by the Ministry of Higher Education, Republic of Cuba. Our special thanks to Rosa Ivette Robles Matos and Yenia Infante Frómeta for their technical assistances. Further, Ana Elisa Bergues Pupo (at present in Max Planck Institute of Colloids and Interfaces, department Theory and Bio-Systems, Potsdam, Germany) thanks Dr. Jesús Manuel Bergues Cabrales (Universidad de San Jorge, Zaragoza, Spain) for his support. We would like to give our special thanks to the Editor in Chief and reviewers of this article for their expert help and invaluable feedback. 


\section{Funding}

This work has been partially supported by the Oriente University, Cuba, under the grants \# 7227 and 7228, Cuba. In addition, Baruch Schneider is partially supported by the Izmir University of Economics. Suleyman Kondakci supports this manuscript. Juan Bory Reyes is partially supported by the ESIMEZacatenco, Instituto Politécnico Nacional (IPN), Mexico, in the framework of SIP programs.

\section{Availability of data and materials}

Data used in this study cannot be shared because our researchers are linked to pharmaceutical and biotechnology Cuban industries by a research contract that includes sensitive experiments. Consequently, this research is under a confidentiality agreement signed for 10 years. Thus, none of the authors of this manuscript may reveal original data. Original data is kept in Bioelectricity group, Research and Innovation Department, National Center of Applied Electromagnetism, Oriente University.

This confidentiality agreement is protected by the Cuban Copyright Law (National Legislature Parliament of the Cuba Republic; Law 14; December 28, 1977; chapters I-III, VII, IX, X), the Invention Law (Official Gazette of the Cuba Republic; Government Decree No. 290; February 1, 2012; pages 9-27; ISSN 1682-7511) and the Work Code includes such inventions under a labor contract (Law 116 of 2014). Besides, Cuban Code of Ethics backs this argumentation.

\section{Authors' contributions}

Study concepts: MMG, JAGJ, LEBC. Study design: MMG, JAGJ, LEBC, AEBP. Data acquisition: MMG, JAGJ, LEBC, AEBP, HMCC, MVJ, MAOM. Quality control of data and algorithms: LEBC. Data analysis and interpretation: MMG, JAGJ, LEBC, AEBP, BS, SK, HMCC, JBR, MVJ, MAOM, TRG, SCAB, JLHC, GVSG. Statistical analysis: MMG, JAGJ, LEBC, AEBP. Manuscript preparation: MMG, JAGJ, LEBC, AEBP, JLHC. Manuscript editing: MMG, LEBC, AEBP. Manuscript review: MMG, JAGJ, LEBC, AEBP, BS, SK, HMCC, JBR, MVJ, MAOM, TRG, SCAB, $J L H C$, GVSG. All authors read and approved the final manuscript.

\section{Competing interests}

The authors declare that they have no competing interests. Patent has not been placed in the Cuban Office of Industrial Property yet (OCPI, Havana, http://www.ocpi.cu) and a priority date is not assigned. As a result, original data and other new results derived of this research cannot be disseminated in any media because this will impede the formal requirement of the world novelty.

\section{Consent for publication}

Not applicable.

\section{Ethics approval}

Ethics committee of the National Center of Applied Electromagnetism approves this study on October 6, 2010. The experiment is run in accord with Good Laboratory Practice Rules and Animal Protection Guidelines of this institution. All procedures on animals are performed in accordance with the EEC Council Directive 86/609 of November 24, 1986; and Guidelines from the Cuban Animal Ethical Committee (Cuban National Bureau of Standards: NC ISO 10993-2). Also, the Cuban Animal Ethical Committee is guided by the EU Directive 2010/63/EU for animal experiments.

\section{Author details}

${ }^{1}$ Pharmacy Department, Oriente University, Natural Science Faculty, Patricio Lumumba Street, Santiago de Cuba 90500, Cuba. ${ }^{2}$ National Center of Seismology Research, Street 7 \# 2 between L and M, Terraza, Santiago de Cuba 90400, Cuba. ${ }^{3}$ Research and Innovation Department, Oriente University, National Center of Applied Electromagnetism, Ave. Las Américas, Santiago de Cuba 90400, Cuba. ${ }^{4}$ Physics Department, Oriente University, Natural Sciences Faculty, Patricio Lumumba Street, Santiago de Cuba 90500, Cuba. ${ }^{5}$ Faculty of Sciences and Literature, Department of Mathematics, Izmir University of Economics, Izmir 353300, Turkey. ${ }^{6}$ Faculty of Engineering and Computer Sciences, Department of Computer Engineering, Izmir University of Economics, Izmir 353300, Turkey. ${ }^{7}$ ESIME-Zacatenco, Instituto Politécnico Nacional, México, DF 07738, Mexico. ${ }^{8}$ Infantil Sur Hospital, Onco-Hematology Department, Santiago de Cuba 90200, Cuba. ${ }^{9}$ Conrado Benítez Oncological Hospital, Mastology Department, Santiago de Cuba 90500, Cuba. ${ }^{10}$ Dirección Municipal de Salud Pública, Servicio de Genética, Santiago de Cuba 90500,
Cuba. ${ }^{11}$ Hospital Provincial Saturnino Lora, Servicio de Medicina Interna, Santiago de Cuba 90500, Cuba. ${ }^{12}$ Facultad de Ciencias Médicas "Diez de Octubre", Universidad de Ciencias Médicas de La Habana, Avenida Independencia No. 8126, Esquina a Calle 100, Boyeros, La Habana, Cuba. ${ }^{13}$ Grupo de las Industrias Biotecnológicas y Farmacéuticas (BioCubafarma), Havana, Cuba.

Received: 12 July 2016 Accepted: 24 February 2017

Published online: 07 March 2017

\section{References}

1. García AF, Mirabal CC, Guevara MAL, Fernández JL, Pérez E. Sickle cell disease painful crisis and steady state differentiation by proton magnetic resonance. Hemoglobin. 2009;33:206-13.

2. Cabrales LEB, Nava JJG, Aguilera AR, Joa JAG, Ciria HMC, González MM, et al. Modified Gompertz equation for electrotherapy murine tumor growth kinetics: Predictions and new hypotheses. BMC Cancer. 2010;10:589.

3. Mckellar R, Lu X. Primary Models. In: Modeling microbial responses in foods. New York: CRC Press, Boca Raton; 2004. p. 21-62.

4. Avrami M. Kinetics of phase change. I. General theory. J Chem Phys. 1939;7:1103-12

5. Avrami M. Kinetics of phase change. II Transformation-time relations for random distribution of nuclei. J Chem Phys. 1940;8:212-24.

6. Workman P, Balmain A, Hickman JA, McNally NJ, Mitchison NA, Pierrepoint CG, et al. UKCCCR guidelines for the welfare of animals in experimental neoplasia. Special Report. Br J Cancer. 1998;58:109-13.

7. Eckenwiler D, Feinholz C, Ells T, Schonfeld T. The declaration of Helsinki through a feminist lens. Int J Feminist Appr Bioethics. 2008;1:161-77.

8. Vaidya VG, Alexandro FJ. Evaluation of some mathematical models for tumor growth. J Biomed Comput. 1982;13:19-35.

9. Marušic M, Bajzer Ž, Vuk-Pavlovic S. Tumor growth in vivo and as multicellular spheroids compared by mathematical models. Bull Math Biol. 1994:56:617-31.

10. Marušic M. Mathematical models of tumor growth. Math Commun 1996:1:175-92.

11. Miklavčič D, Jarm T, Karba R, Serša G. Mathematical modelling of tumor growth in mice following electrotherapy and bleomycin treatment. Math Simul Comp. 1995;39:597-602.

12. Bao-Quan A, Xian-Ju W, Guo-Tao L, Liang-Gang L. Correlated noise in a logistic growth model. Phys Rev E. 2003;67:022903.

13. Jiménez RP, Hernández EO. Tumor-host dynamics under radiotherapy. Chaos, Solitons Fractals. 2011:44:685-92.

14. Cabrales LEB, Aguilera AR, Jiménez RP, Jarque MV, Mateus MAO, Ciria HMC, et al. Mathematical modeling of tumor growth in mice following low-level direct electric current. Math Simul Comp. 2008;78:112-20.

15. Ferrante L, Bompadre S, Possati L, Leone L. Parameter estimation in a Gompertzian stochastic model for tumor growth. Biometrics. 2000;56:1076-81.

16. Rofstad EK, Graff BA. Thrombospondin-1-mediated metastasis suppression by the primary tumor in human melanoma xenografts. J Invest Dermatol. 2001;117:1042-49.

17. Cotran RS, Kumar V, Collins T. Patología Estructural y Funcional. 6th ed. S.A. U. Madrid: McGraw-Hill-Interamericana de España; 1999.

18. Schuster $\mathrm{R}$, Schuster $\mathrm{H}$. Reconstruction models for the Ehrlich Ascites Tumor for the mouse. Mathematical Population Dynamics. 1995;2:335-48.

19. Prajneshu. Environmental stochasticity of Gompetz model with time delay. Comm Statist - Theory Methods. 1983;12:1359-69.

20. Murray JD. Mathematical Biology, An introduction. New York: Springer; 2002

21. Waliszewski P, Konarski J. The Gompertzian curve reveals fractal properties of tumor growth. Chaos, Solitons and Fractals. 2003:16:665-74.

22. Davies PCW, Demetrius L, Tuszinsky JA. Cancer as a dynamical phase transition. Theor Biol Med Model. 2011:8:30.

23. Paszek MJ, Zahir N, Jonhson KR, Lakins JN, Rozenberg Gl, Gefen A, et al. Tensional homeostasis and the malignant phenotype. Cancer Cell. 2005;8:241-54.

24. Fuhrmann A, Staunton JR, Nandakumar V, Banyai N, Davies PCW, Ros R. AFM stiffness nanotomography of normal, metaplastic and dysplastic human esophageal cells. Phys Biol. 2011:8:015007.

25. Zhang Q, Austin RH. Physics of cancer: the impact of heterogeneity. Annu Rev Condens Matter Phys. 2012;3:363-82.

26. Wang J, Kou HC, Gu XF, Li JS, Xing LQ, Hu R, et al. On discussion of the applicability of local avrami exponent: errors and solutions. Materials Lett. 2009;63:1153-55. 
27. Liu F, Sommer F, Bos C, Mittemeijer EJ. Analysis of solid state phase transformation kinetics: models and recipes. Inter Mat Rev. 2007:52:193-212.

28. Kooi BJ. Monte Carlo simulations of phase transformations caused by nucleation and subsequent anisotropic growth: extension of the johnsonmehl-avrami-kolmogorov theory. Phys Rev B. 2004;70:224108.

29. Maragoni AG, Wesdorp LH. Structure and Properties of Fat Crystal Networks. 2nd ed. Florida: CRC Press Taylor and Francis Group; 2004.

30. Aloev VZ, Kozlov GV, Zaikov GE. Relationship between the exponent of the Kolmogorov-Avrami equation and the fractal dimension in the crystallisation of uniaxially stretched crosslinked polychloroprene. Int Polym Sci Tech. 2004;31:33-5.

31. Markworth AJ, Lawrence GM. Kinetics of anisothermal phase transformations. J Appl Phys. 1983;54:3502-08.

32. Press WH, Teukolsky SA, Vetterling WT, Flannery BP. Modeling of Data, in Numerical Recipes in C, The Art of Scientific Computing. 2nd ed. New York: Cambridge University Press; 1992

33. Benzekry S, Lamont C, Beheshti A, Tracz A, Ebos JML, Hlatky L, et al. Classical mathematical models for description and prediction of experimental tumor growth. PLoS Comput Biol. 2014;10:e1003800.

34. Laird AK. Dynamics of tumour growth: comparison of growth rates and extrapolation of growth curve to one cell. Br J Cancer. 1965;19:278-91.

35. Brú A, Albertos S, Subiza JL, García-Asenjo JL, Brú I. The universal dynamics of tumor growth. Biophys J. 2003;85:2948-61.

36. Chaplain MAJ. Avascular growth, angiogenesis and vascular growth in solid tumours: the mathematical modeling of the stages of tumor development. Math Comp Model. 1996;23:47-87.

37. Hogea CS, Murray BT, Sethian JA. Simulating complex tumor dynamics from avascular to vascular growth using a general level set method. J Math Biol. 2006;53:86-134

38. Drasdo D, Höhme S. A single-cell-based model of tumor growth in vitro: monolayers and spheroids. Phys Biol. 2005;2:133.

39. Izquierdo-Kulich E, Alonso-Becerra E, Nieto-Villar JM. Entropy production rate for avascular tumor growth. J Modern Phys. 2011;2:615

40. Witten TA, Sander LM. Diffusion limited aggregation, a kinetic critical phenomenon. Phys Rev Lett. 1981;47:1400.

41. Roose T, Chapman SJ, Maini PK. Mathematical models of avascular tumor growth. SIAM Rev. 2007:49:179-208

42. Haltiwanger S. The Electrical Properties of Cancer Cells. 2008. http://www. royalrife.com/haltiwanger1.pdf. Accessed 15 Apr 2016.

43. Byrne HM, Chaplain MAJ. Modelling the role of cell-cell adhesion in the growth and development of carcinomas. Math Comp Model. 1996;24:1-17.

44. Mandelbrot B. Fractals, Form, Chance, and Dimension. San Francisco: Freeman; 1977.

45. Waliszewski P, Konarski J. A mystery of the Gompertz function. In: Losa GA Merlini D, Nonnenmacher TF, Weibel ER, editors. Fractals in Biology and Medicine, Mathematics and Biosciences in Interaction Books. Germany: Birkhäuser Basel, Springer; 2005. p. 277-86.

46. Shim EB, Kim YS, Deisboeck TS. Analyzing the dynamic relationship between tumor growth and angiogenesis in a two dimensional finite element model. 2007. arXiv:q-bio/0703015v1 [q-bio.TO]. Accessed 10 February 2016

47. Leibovitz A. Development of media for isolation and cultivation of human cancer cells. In: Fogh J, editor. Tumor cells in vitro. New York: Springer Science + Business Media, LLC; 1975. p. 23-50.

48. Molski M, Konarski J. Coherent states of gompertzian growth. Phys Rev E. 2003;68:0219161-7.

49. Molski M. Biological growth in the fractal space-time with temporal fractal dimension. Chaotic Model Simul. 2012;1:169-75.

50. de Arruda PFF, Gatti M, Junior FNF, de Arruda JGF, Moreira RD, Junior LOM, et al. Quantification of fractal dimension and Shannon's entropy in histological diagnosis of prostate cancer. BMC Clin Pathol. 2013;13:6.

51. Plankar M, Jerman I, Krašovec R. On the origin of cancer: Can we ignore coherence? Prog Biophys Mol Biol. 2011;106:380-90.

52. Izquierdo-Kulich E, Regalado O, Nieto-Villar JM. Fractal origin of the Gompertz equation. Rev Cub Fis. 2013:30:26.

53. Sekino M, Ohsaki H, Yamaguchi-Sekino S, Iriguchi N, Ueno S. Low-frequency conductivity tensor of rat brain tissues inferred from diffusion MRI. Bioelectromagnetics. 2009;30:489-99.

54. Hanahan D, Weinberg RA. Hallmarks of cancer: the next generation. Cell. 2011:144:646-74.
55. Stylianopoulous T, Martin JD, Snuderl M, Mpekris F, Jain SR, Jain RK. Coevolution of solid stress and interstitial fluid pressure in tumors during progression: Implications for vascular collapse. Cancer Res. 2013;73:3833-41.

56. Guha E. Basic Thermodynamics. India: Alpha Science International Ltd; 2000.

57. Sciumé $G$, Shelton $S$, Grat WG, Miller $C$, Hussain F, Ferrari M, et al. A multiphase model for three-dimensional tumor growth. New J Phys. 2013;15:015005.

58. Pardoll DM. The blockade of immune checkpoints in cancer immunotherapy. Nat Rev Cancer. 2012;12:252-64.

\section{Submit your next manuscript to BioMed Central and we will help you at every step:}

- We accept pre-submission inquiries

- Our selector tool helps you to find the most relevant journal

- We provide round the clock customer support

- Convenient online submission

- Thorough peer review

- Inclusion in PubMed and all major indexing services

- Maximum visibility for your research

Submit your manuscript at www.biomedcentral.com/submit
C) Biomed Central 